\title{
Trapping Biological Species in a Lab-on-Chip Microsystem: Micro Inductor Optimization Design and SU8 Process
}

\author{
Christophe Escriba $^{1,2}$, Rémy Fulcrand ${ }^{1,2}$, Philippe Artillan ${ }^{1,2}$, David Jugieu ${ }^{1,2}$, \\ Aurélien Bancaud $^{1,2}$, Ali Boukabache $e^{1,2}$, Anne-Marie Gue ${ }^{1,2}$, \\ and Jean-Yves Fourniols ${ }^{1,2}$ \\ ${ }^{1}$ LAAS-CNRS ; Université de Toulouse ; 7, avenue du Colonel Roche, F-31077 Toulouse, \\ France \\ ${ }^{2}$ Université de Toulouse; UPS, INSA, INP, ISAE
}

\begin{abstract}
Micro-spiral inductor dedicated to microbeads manipulation in a fluidic channel had been optimized by analytical modelling correlated to multi physics fem numerical Maxwell@3D L software. Main advantage of the analytical model described below is time analysis calculus decrease of and the capability offered to optimize geometrical and electrical parameters of the inductor. First experimental results show a good correlation between simulation and realized integrating micro-devices in a fluidic channel.
\end{abstract}

Keywords: Analytical model, Finite element method, Magnetic field / force, Magnetic actuators, magnetic bead separation.

\section{Introduction}

Lab-On-Chip micro-systems with embedded analysis are the two major concepts of MEMS dedicated to fluidic applications. In order to realize biochemical analysis or pharmacological screening, separation of biological species, we propose magnetic bead as a very useful technique. In most cases, the magnetic activation is macroscopic and positioned outside of the system. As these approaches limits strongly the MEMS integrating process, we develop in LAAS-CNRS lab, a multi-functional magnetic source in order to realize a complete fluidic micro-system for handling paramagnetic beads.

By reporting here first works dedicated to model the behaviour of magnetic sources and the forces generated on microbeads, we present two methods allowing to determine magnetic field generated by a planar inductor: one entirely analytical and other by using the multi-physics software Maxwell@3D. Comparisons are discussed in terms of performances, accuracy, and CPU time computation. As a complementary part, we present first technological results of research (in progress) in order to integrate a family of inductors in channels. These preliminary devices confirm the feasibility of our approach. 


\section{Determination of Magnetic Field Distribution in a 3D Space. Analytical Modeling}

The magnetostatic field induced by a DC current flowing in an electric conductor is given by the following two Maxwell's equations:

$$
\begin{aligned}
\nabla \times \vec{H} & =\vec{J} \\
\nabla \cdot \vec{B} & =0
\end{aligned}
$$

With the following constitutive (material) relationship being also applicable:

$$
\vec{B}=\mu_{0} \mu_{r} \vec{H}+\mu_{r} \overrightarrow{M_{p}}
$$

- $H(x, y, z)$ is the magnetic field strength

- $B(x, y, z)$ is the magnetic flux density.

- $J(x, y, z)$ is the conduction current density.

- $\operatorname{Mp}(x, y, z)$ is the permanent magnetization.

- $\mu_{0}=4 * \pi * 10^{-7} H \cdot m^{-1}$ is the permeability of vacuum.

- $\mu_{r}$ is the relative permeability.

First, we consider all the segments constituting the inductor which coordinates are $\left[\alpha_{\mathrm{i}} \omega_{\mathrm{i}}\right]$ in $\Re$ frame (Figure 1 ).

Geometrical and electrical characteristics are:

- $\mathrm{L}_{1} \quad$ : length to the first segment of the inductor (axis $\mathrm{Ox}$ )

- $\mathrm{L}_{2} \quad$ : length to the second segment of the inductor (Oy)

- $\mathrm{N} \quad$ : number of spires,

- $\quad \mathrm{s}$ : inter-coil distance,

- I : current in all segments

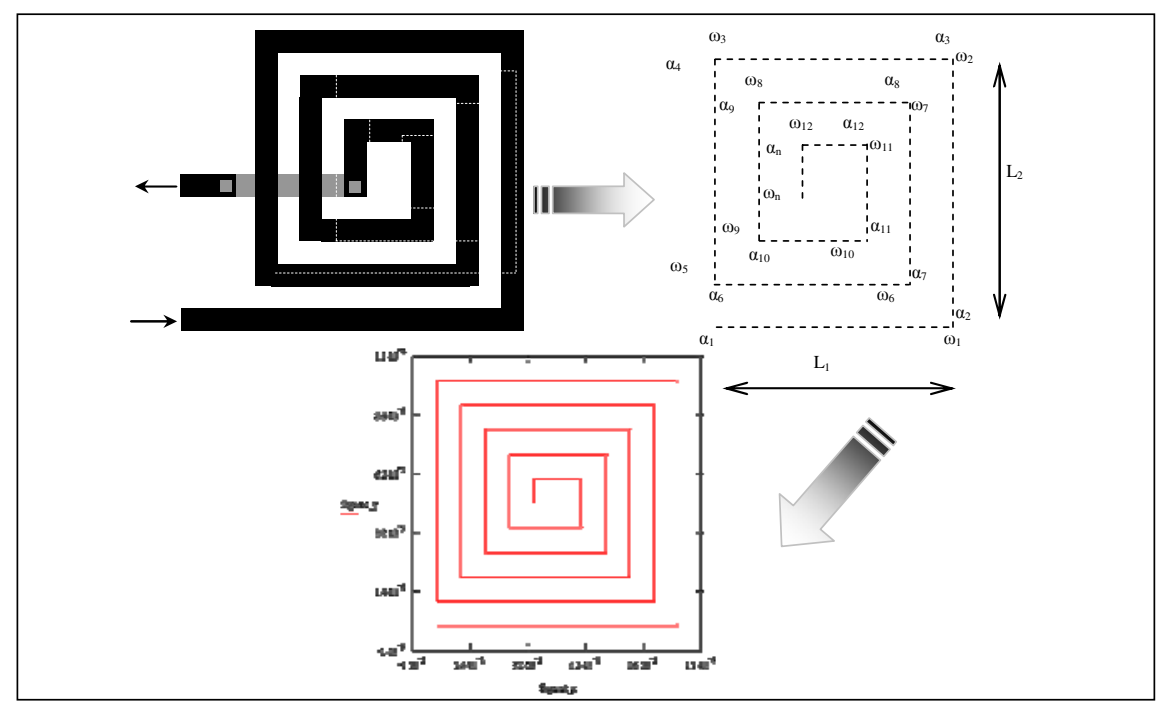

Fig. 1. Equivalent topology of planar inductor and display results for five spires inductor 
In order to evaluate quickly magnetic field generated by the induction coil, we consider the inductor in frame $\Re$ like a set of elementary segments where the magnetic field $B$ is first obtained for each segment $\left[\alpha_{i} \omega_{i}\right]$, and the total magnetic field will be determined by summation of each elementary field calculated in the frame $\Re^{\prime}$

A. STEP1: Magnetic field expression $\vec{B}\left(x^{\prime}, y^{\prime}, z^{\prime}\right)$ created by a single segment in $\Re^{\prime}$

We consider $M$ point, included in the (x0y) area where we define $[\alpha, \omega]$ segment. We define the orthonormal frame $\Re^{\prime}$ with $\alpha$ origin, and first axis [ $\left.\alpha, \omega\right]$, Figure 2.

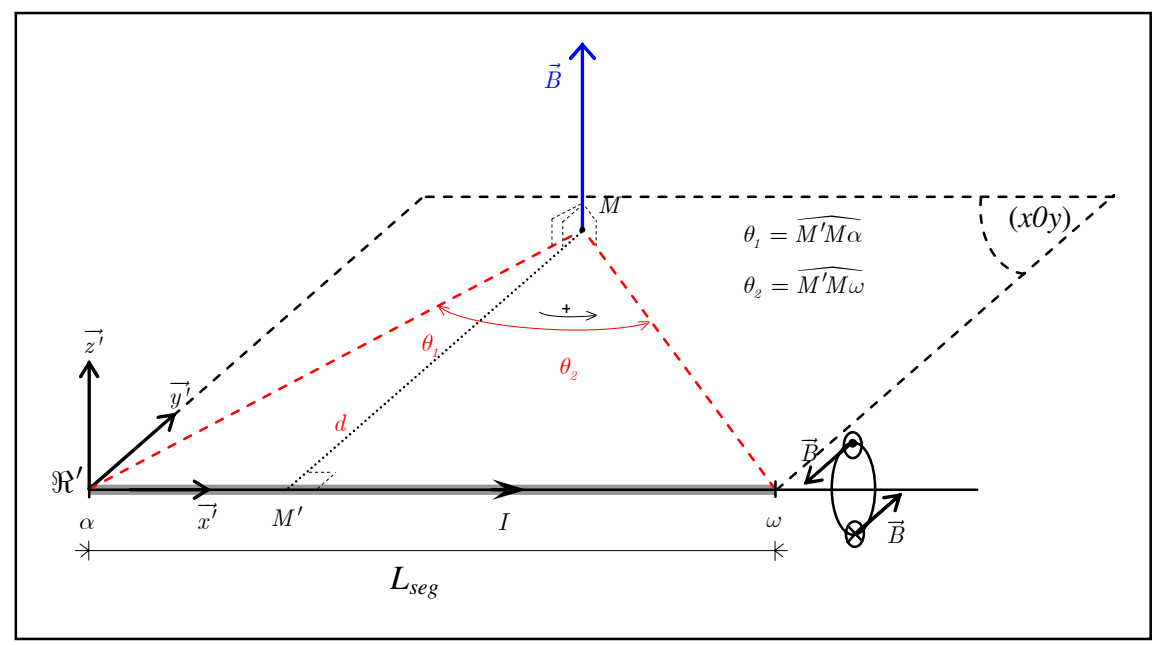

Fig. 2. Magnetic field created by the $[\alpha \omega]$ segment at point $M$

Distance $d$ between point $M$ and $[\alpha, \omega]$ segment is evaluated by the equation:

$$
d=\sqrt{y^{2}+z^{2}}
$$

Using $\theta_{2}, \theta_{1}$ angles defined in Figure 2, the magnetic field $\left.\vec{B}\left(x^{\prime}, y^{\prime}, z^{\prime}\right)\right|_{\Re^{\prime}}$ in $\Re^{\prime}$ frame is:

$$
\left.B\left(x^{\prime}, y^{\prime}, z^{\prime}\right)\right|_{\Re^{\prime}}=\frac{\mu_{0} I}{4 \pi d}\left[\sin \theta_{1}-\sin \theta_{2}\right] \overrightarrow{z^{\prime}}
$$

where $\theta_{1}=-\tan ^{-1}\left(\frac{x}{d}\right)$ and $\theta_{2}=\tan ^{-1}\left(\frac{L_{\text {seg }}-x}{d}\right)$

B. STEP2: Summation of all magnetic field $\vec{B}$ segments

The $\Re^{\prime}$ frame is obtained by a translation of $\mathrm{T}$ vector and a linear transformation through $[\mathrm{P}]$ matrix (Figure 3). 


$$
\left.\overrightarrow{O M}\right|_{\Re}=\left.\vec{T}\right|_{\Re}+\left.[P] \cdot \overrightarrow{\alpha M}\right|_{\Re}
$$

With:

$$
\vec{T}=\overrightarrow{O \alpha}, \overrightarrow{x^{\prime}}=\frac{\overrightarrow{\alpha \omega}}{\|\overrightarrow{\alpha \omega}\|}, \overrightarrow{z^{\prime}}=\frac{\overrightarrow{\alpha \omega} \times \overrightarrow{\alpha M}}{\|\overrightarrow{\alpha \omega} \times \overrightarrow{\alpha M}\|}, \overrightarrow{y^{\prime}}=\overrightarrow{z^{\prime}} \times \overrightarrow{x^{\prime}}
$$

Where $(x$ is vector product).

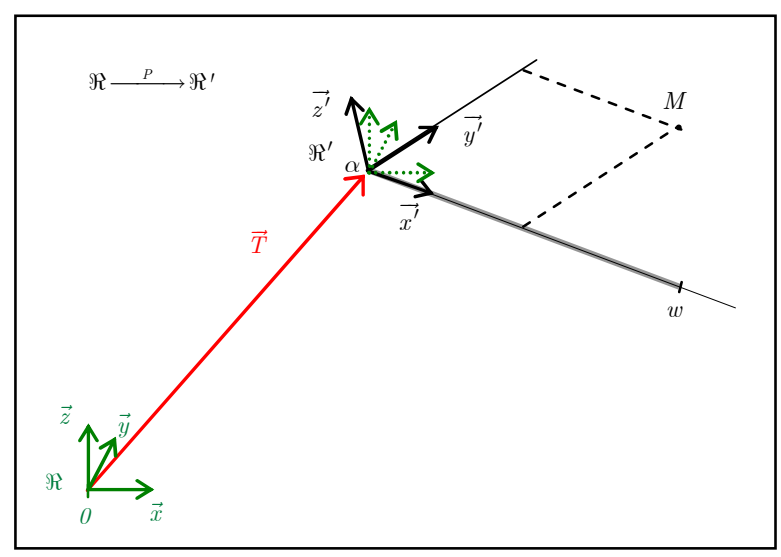

Fig. 3. $\Re \leftrightarrow \Re^{\prime}$ frame definitions

Combining $\mathrm{M}$ coordinates with $\alpha$ and $\omega$ points, we obtain scalar elements of [P] matrix by concatenation of $\overrightarrow{x^{\prime}}, \overrightarrow{y^{\prime}}, \overrightarrow{z^{\prime}}$ :

$$
P=\left[\overrightarrow{x^{\prime}}\left|\overrightarrow{y^{\prime}}\right| \overrightarrow{z^{\prime}}\right]
$$

M point coordinates are showing in $\Re$ frame. For calculus of every components of $B_{i}$, it's required to transfer this coordinates in $\Re^{\prime}$ according to (Figure 4):

$$
\left.\overrightarrow{\alpha_{i} M}\right|_{\Re_{i}{ }^{\prime}}=P^{T} \cdot\left(\left.\overrightarrow{O M}\right|_{\Re}-\left.\vec{T}\right|_{\Re}\right)
$$

Considering, $\left.\overrightarrow{B_{i}}\right|_{\Re}=\left.P \cdot \overrightarrow{B_{i}}\right|_{\Re_{i}}$, magnetic field associated to $\left[\alpha_{i}, \omega_{i}\right]$ segment, the total magnetic field is obtain by summation of every $B_{i}$ components:

$$
\left.\overrightarrow{B_{t o t}}\right|_{\Re}=\left.\sum_{i=1}^{N_{s e g}} \overrightarrow{B_{i}}\right|_{\Re}
$$




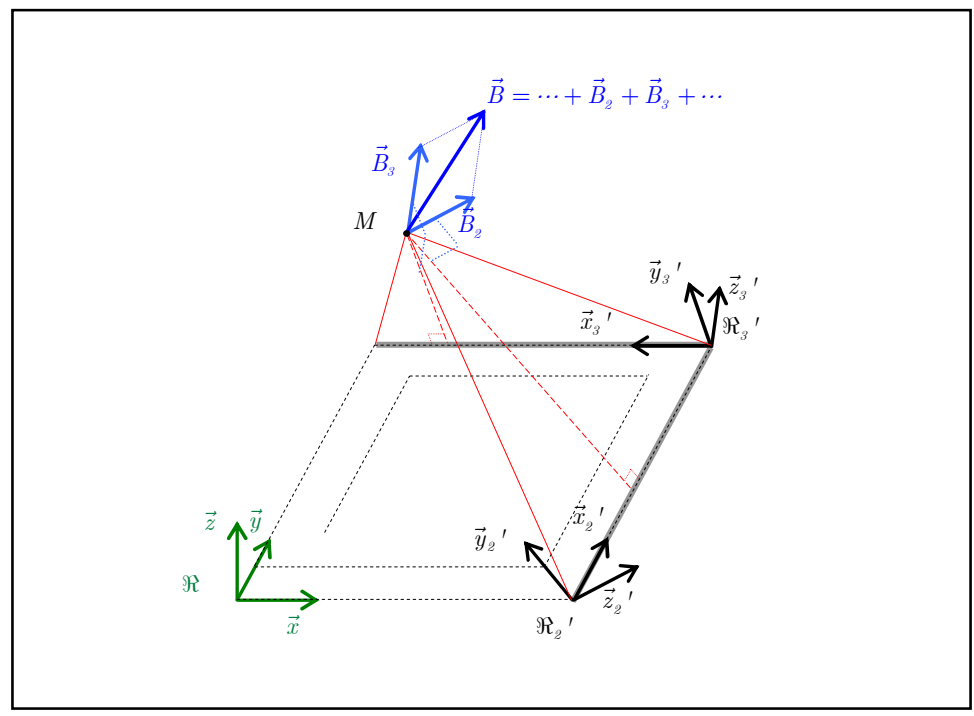

Fig. 4. Coordinates systems describing the micro-inductor and the global magnetic field at M point

\section{Estimation of magnetic field of planar induction}

Figure 5, represents the magnetic field computed with our algorithm, for a inductor with 5 spires, side $100 \mu \mathrm{m}$ by $100 \mu \mathrm{m}$ covered by an electrical current $\mathrm{I}=5 \mathrm{~mA}$ at high $\mathrm{z}=50 \mu \mathrm{m}$.

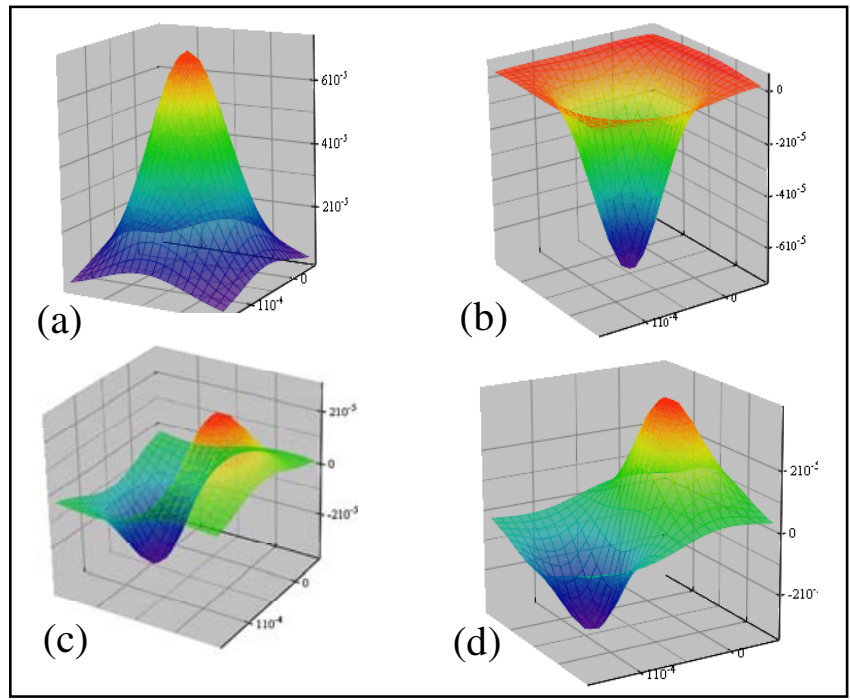

Fig. 5. Magnetic field computed: (a) modulus; (b) along $\mathrm{Oz}$ axis, (c) along Ox axis, (d) along Oy axis 
We can observe that the $\mathrm{z}$ component present an extrema above the center of the inductor and a value close to zero along $\mathrm{Ox}$ and $\mathrm{Oy}$.

\section{Validation of the Analytical Approach}

In order to check the validity of the previous analytical modelling, we compare results obtained width the Maxwell ${ }^{\circledR} 3 \mathrm{D}$ software and hence a FEM approach with those obtained with our model.

The electric field is restricted to the objects modelled as real (non ideal) conductors and is totally decoupled from the magnetic field. We consider that there no time variation effects and objects are considered to be stationary. So previously, the magnetostatic field solution verifies the Maxwell's equations. The MAXWELL3D magnetostatic solver considers the magnetic field $\mathrm{H}$ with the following components:

$$
\overrightarrow{\mathrm{H}}=\overrightarrow{\mathrm{H}_{\mathrm{p}}}+\nabla \varphi+\overrightarrow{\mathrm{H}_{\mathrm{c}}}
$$

Where $\varphi$ is the magnetic scalar potential, $\overrightarrow{\mathrm{H}_{\mathrm{p}}}$ is a particular solution constructed by assigning values to all the edges in the mesh in such a way that Ampere's law holds on all contours of all tetrahedral faces in the mesh, and $\overrightarrow{\mathrm{H}_{\mathrm{c}}}$ accounts for permanent magnet if any. Thus, the Degrees Of Freedom (DOFs) are the nodal values of the magnetic scalar potential with ten values per tetrahedron at each of the four vertices and all six mid edge nodes, ensuring a quadratic approximation inside each finite element.

There are major advantages of this formulation over other existing ones, including using considerably fewer computational resources (due to the scalar nature of the DOFs), not requiring a gauge due to excellent numerical stability, significantly reducing cancellation errors, and capably of automatically multiplying connected iron regions. The magnetostatic solver handles both 3D linear and nonlinear problems. The magnetostatic solver calculates the magnetic field distribution produced by a combination of known DC current density vector distribution (Figure 6).

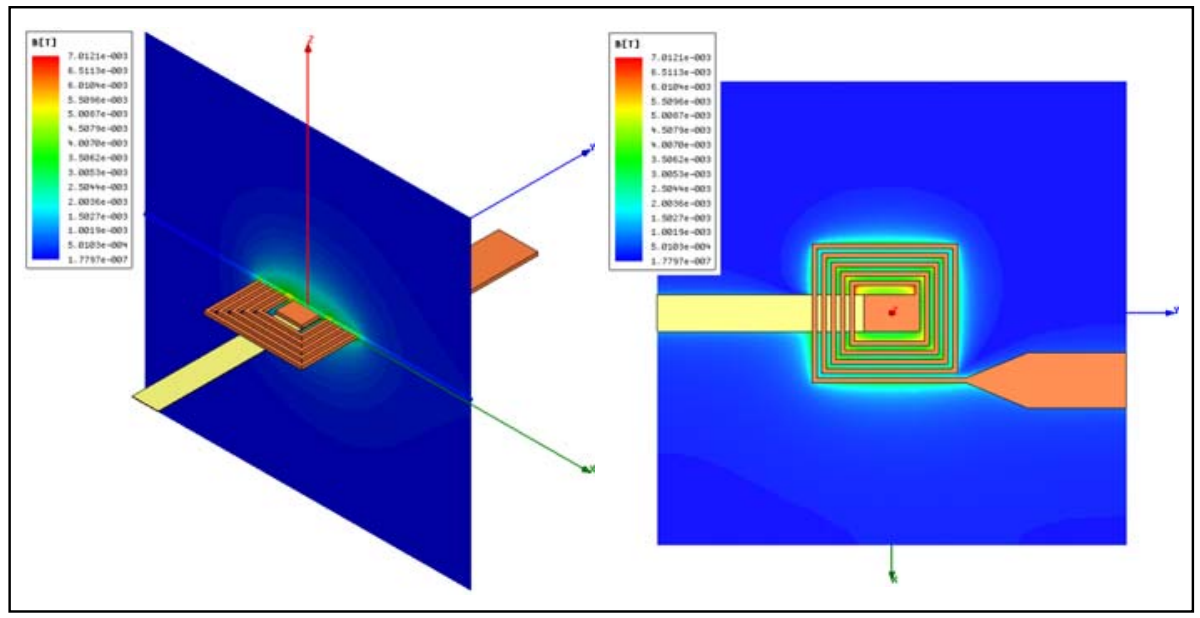

Fig. 6. 3D representation of the magnetic field. a) in $(X, Z)$ plan and $b)$ in $(X, Y)$ plan 


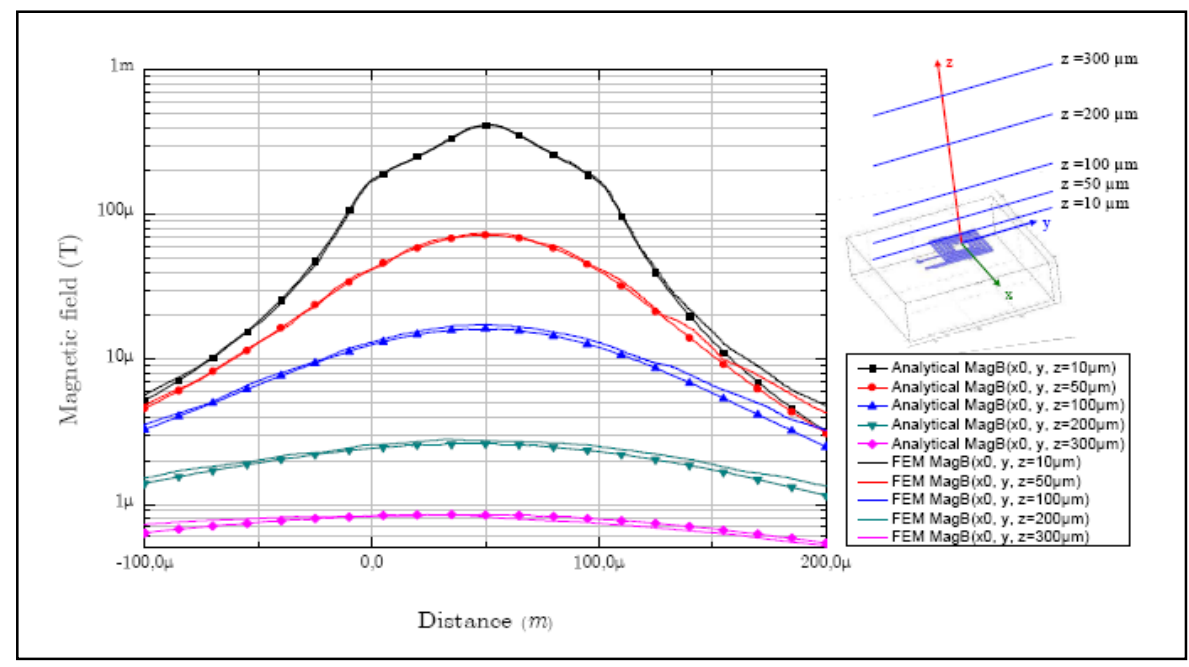

Fig. 7. Magnetic field calculated by analytical and finite element methods

Various coil designs have been simulated with the use of MAXWELL3D. For example, Figure 6 shows the magnetic field calculated for a micro-coil with 5 turns, a conductor square section of $5 \mu \mathrm{m} \times 5 \mu \mathrm{m}$ and a $50 \mathrm{~mA}$ current.

The comparison between analytical and FEM modelling of magnetic field $\vec{B}$ is detailed in Figure 7, where we have reported the values of B calculated at different heights $\mathrm{z}$ from the coil.

These results confirm the good correlation between the two methods and fully validate the efficiency of the analytical model. Thus we can assume that the analytical model developed is powerful for parametric optimization.

\section{Application: Magnetic Beads Trapping}

Using our analytical model, we have optimized the design of an integrated magnetic actuator dedicated to the manipulation of microbeads in lab on chip devices. The criterion of optimization was to obtain magnetic force allowing the trapping super paramagnetic microbeads in micro-channels. The magnetic forces have been calculated starting from the simulation results. For this purpose, we have integrated some micro-inductors in a micro-fluidic device made entirely with a biocompatible SU-8 polymer. This prototype will undergo a series of characterization and validation.

\section{Magnetic Force Exerted on a Magnetic Microbead}

The force $\vec{F}_{p}^{m}$ exerted by a magnetic field on a particle is represented by the gradient of the magnetic interaction energy of the particle immersed in the magnetic field. 


$$
\overrightarrow{\mathrm{F}}_{\mathrm{p}}^{\mathrm{m}}=-\nabla \overrightarrow{\mathrm{U}}_{\mathrm{p}}^{\mathrm{m}}
$$

The magnetic energy can be expressed by:

$$
\overrightarrow{\mathrm{U}}_{\mathrm{p}}^{\mathrm{m}}=-\frac{1}{2} \mu_{0} \int \overrightarrow{\mathrm{M}}_{\mathrm{p}} \cdot \overrightarrow{\mathrm{H}} \cdot \mathrm{dv}
$$

Where $\mu_{0}=4 * \pi * 10^{-7} H \cdot m^{-1}$ is the vacuum permeability and $\vec{M}_{p}$ the magnetizing of this particle by the magnetic field of excitation $\vec{H}$.

In the case of a particle with very small dimension, the integration of (13) is then replaced by the value of the field in the center of the particle multiplied by the volume $V_{p}$ of this particle:

$$
\overrightarrow{\mathrm{U}}_{\mathrm{p}}^{\mathrm{m}}=-\frac{1}{2} \mathrm{~V}_{\mathrm{p}} \mu_{0} \overrightarrow{\mathrm{M}}_{\mathrm{p}} \cdot \overrightarrow{\mathrm{H}}
$$

In air or the vacuum, a diamagnetic or paramagnetic micro particle acquires a magnetizing $\vec{M}_{p}$ which a function of the magnetic susceptibility $\chi_{p}$, the field of excitation $\vec{H}$ and the demagnetization coefficient $\mathrm{D}(\mathrm{D}=1 / 3$ for a sphere, $\mathrm{D}=1$ for a parallelepiped, $\mathrm{D}=0$ long bar).

$$
\overrightarrow{\mathrm{M}}_{\mathrm{p}}=\frac{\chi_{\mathrm{p}}}{1+\chi_{\mathrm{p}} \cdot \mathrm{D}} \overrightarrow{\mathrm{H}}
$$

It is considered that $1+\chi_{p} . D \approx 1$ and equation (15) can be simplified:

$$
\overrightarrow{\mathrm{M}}_{\mathrm{p}}=\chi_{\mathrm{p}} \overrightarrow{\mathrm{H}}
$$

Equation (14) becomes then:

$$
\overrightarrow{\mathrm{U}}_{\mathrm{p}}^{\mathrm{m}}=-\frac{1}{2} \mathrm{~V}_{\mathrm{p}} \mu_{0} \chi_{\mathrm{p}}|\mathrm{H}|^{2}
$$

The expression of the magnetic force deduced from (12) and (17) is given by:

$$
\overrightarrow{\mathrm{F}}_{\mathrm{p}}^{\mathrm{m}}=\mu_{0} \mathrm{~V}_{\mathrm{p}} \chi_{\mathrm{p}}(\overrightarrow{\mathrm{H}} . \nabla) \overrightarrow{\mathrm{H}}
$$

This expression shows that direction of the force does not depend on the field sign but of the product of field with its gradient. Its orientation is also related to the susceptibility of the particle. The total magnetic force exerted on the particle is given by:

$$
\overrightarrow{\mathrm{F}}_{\text {MagTtl }}=\overrightarrow{\mathrm{F}}_{\mathrm{p}}^{\mathrm{m}}+\overrightarrow{\mathrm{F}}_{\mathrm{f}}^{\mathrm{m}}=\mu_{0} \mathrm{~V}_{\mathrm{p}}\left(\chi_{\mathrm{p}}-\chi_{\mathrm{f}}\right)(\overrightarrow{\mathrm{H}} \cdot \nabla) \overrightarrow{\mathrm{H}}
$$

Because of very low value of susceptibility of the immersing medium $\left(\chi_{\mathrm{f}}=-9.048 * 10^{-6}\right.$ for water $)$ we assume that the magnetic field induced by the medium can be neglected and that:

$$
\overrightarrow{\mathrm{B}}=\overrightarrow{\mathrm{B}}_{\text {coil }}=\mu_{0} \overrightarrow{\mathrm{H}}
$$


The equation of the total magnetic force becomes:

$$
\overrightarrow{\mathrm{F}}_{\text {MagTtl }}=\frac{\mathrm{v}_{\mathrm{p}} \chi_{\mathrm{p}}}{\mu_{0}}\left(\overrightarrow{\mathrm{B}}_{\text {coil }} \cdot \nabla\right) \overrightarrow{\mathrm{B}}_{\text {coil }}
$$

The components of the magnetic force in a Cartesian reference mark are expressed by:

$$
\vec{F}_{\text {MagTtl }}=\frac{v_{p}}{\mu_{0}}\left(\chi_{p}\right)\left(\begin{array}{lll}
B_{x} & B_{y} & B_{z}
\end{array}\right)\left(\begin{array}{c}
\frac{d}{d x} \\
\frac{d}{d y} \\
\frac{d}{d z}
\end{array}\right)\left(\begin{array}{lll}
B_{x} & B_{y} & B_{z}
\end{array}\right)
$$

Thus in the case of a super paramagnetic particle in a conveying fluid like water, the particle is attracted towards magnetic field maxima.

By convention, the magnetic actuator (coils) being in $(\mathrm{X}, \mathrm{Z})$ plan, the force is taken into account only in the (X, Z) plan (Figure 8) and:

$$
\begin{aligned}
& \mathrm{F}_{\text {MagTtlx }}(\mathrm{x}, \mathrm{z})=\frac{\mathrm{V}_{\mathrm{p}}}{\mu_{0}}\left(\chi_{\mathrm{p}}\right)\left(\mathrm{B}_{\mathrm{x}} \frac{\mathrm{dB}_{\mathrm{x}}}{\mathrm{dx}}+\mathrm{B}_{\mathrm{z}} \frac{\mathrm{dB}_{\mathrm{x}}}{\mathrm{dz}}\right) \\
& \mathrm{F}_{\text {MagTtlz }}(\mathrm{x}, \mathrm{z})=\frac{\mathrm{V}_{\mathrm{p}}}{\mu_{0}}\left(\chi_{\mathrm{p}}\right)\left(\mathrm{B}_{\mathrm{x}} \frac{\mathrm{dB}_{\mathrm{z}}}{\mathrm{dx}}+\mathrm{B}_{\mathrm{z}} \frac{\mathrm{dB}_{\mathrm{z}}}{\mathrm{dz}}\right)
\end{aligned}
$$

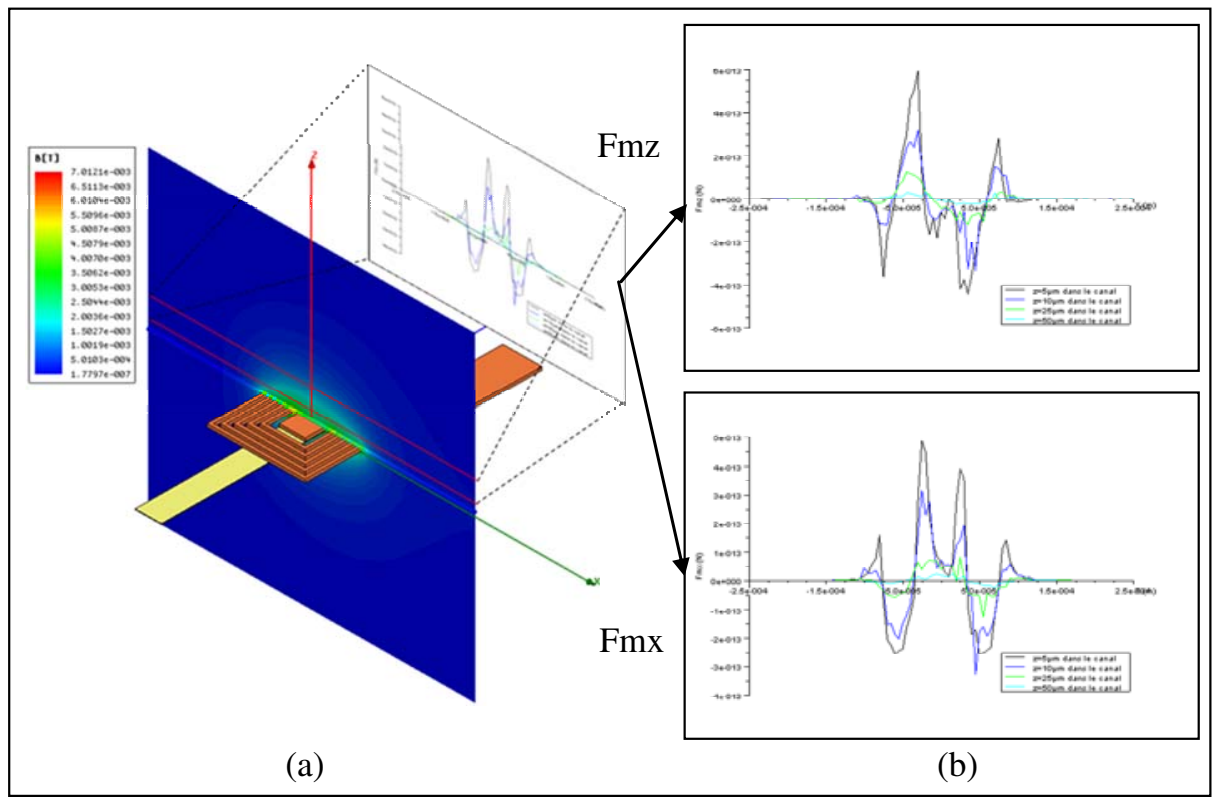

Fig. 8. (a) Sight $3 D$ of the device and the plan $(X, Z)$ where is represented the distribution of the magnetic field B. In red, the fluidic channel where calculations of the forces for different height are localized. (b) Graphic of the magnetic forces according to $\mathrm{X}$ and $\mathrm{Z}$ obtained by the post-processing. 


\section{Technological Realizations}

\subsection{Process Flow for the Fabrication of Integrated Microsystem}

One of the main tasks of this work was to develop a generic polymer based technology in order to manufacture a microfluidic device able to trap functionalized micro beads. As it will be shown below, this technology combines standard electroplating technique and lamination technologies in order to achieve flexible and transparent full polymer systems with the precision and reliability of "stat-of-the-art" microsystems. The requirements for the integrated microsystem are:

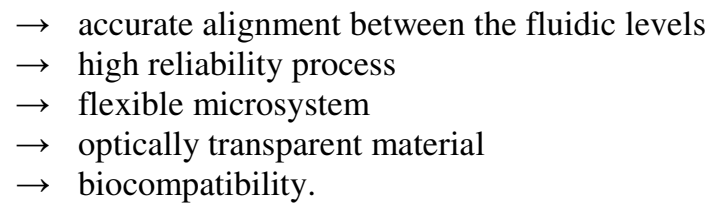

According to these criteria, we have chose to utilize the polyethylene terephthalate layer (PET sheet, for the flexibility) as a "sacrificial" material, the epoxy-based negative photoresist SU-8 on the structural material for the microfluidic network (transparency, biocompatibility, very high aspect ratio...) and copper for the electroplating process of the micro-coil. The technological process flow can be described in Figure 9.

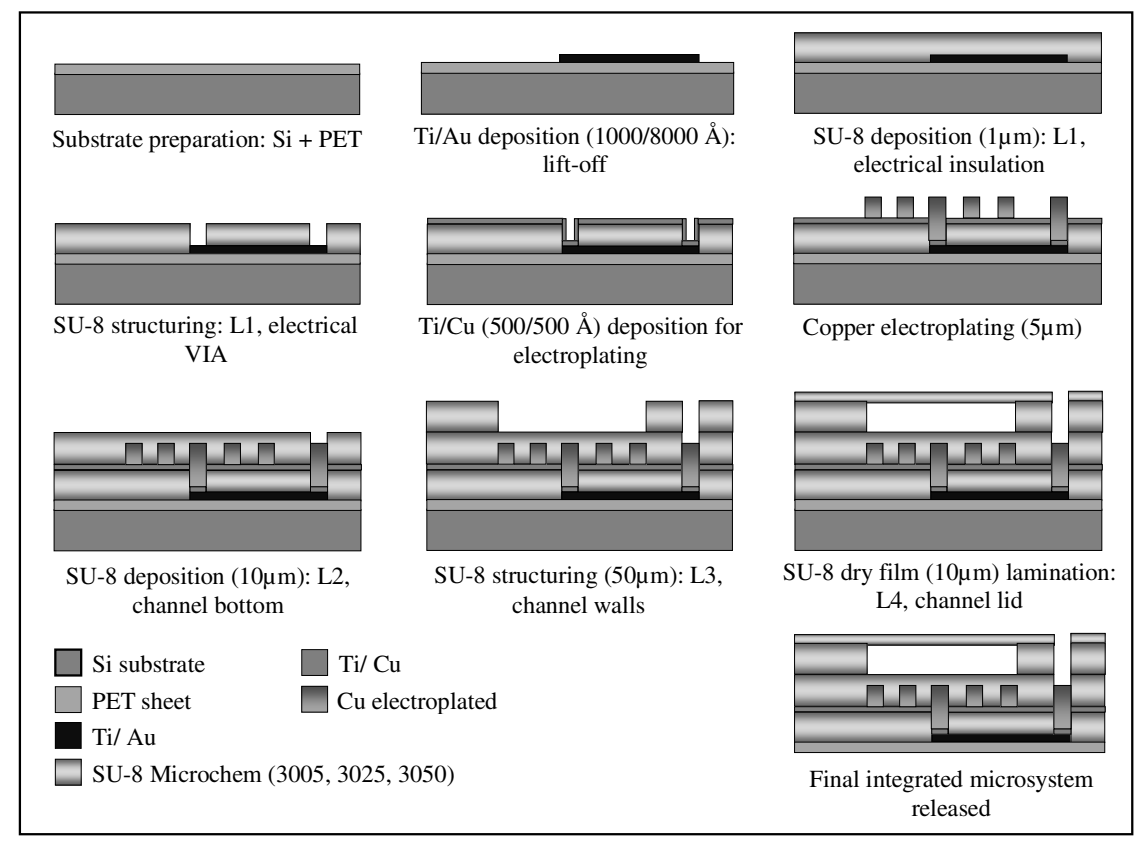

Fig. 9. Process flowchart for the fabrication of the flexible integrated microsystem 


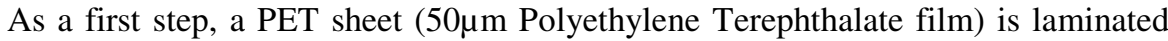
on the top of a silicon wafer. The PET was chosen for its poor adhesion properties and transparency. Then, conductive tracks are patterned (Ti/Au) by lift-off processing (Figure 10 (a)). A layer of SU-8 (Microchem 3005) is deposited on top of the structure in order to perform electrical insulation between conductive tracks and coils, and to create electrical VIA (Figure 10 (b)). A seed layer of $\mathrm{Ti} / \mathrm{Cu}(500 / 500 \AA$ ) is deposited followed by the deposition and patterning of a positive photoresist (AZ4562) in order to create the electroplating mold. Then, copper coils are electroplated $(5 \mu \mathrm{m})$ into the resist mould (Figure $10(\mathrm{c}))$ and the photoresist and seed layers $(\mathrm{Ti} / \mathrm{Cu})$ are removed.

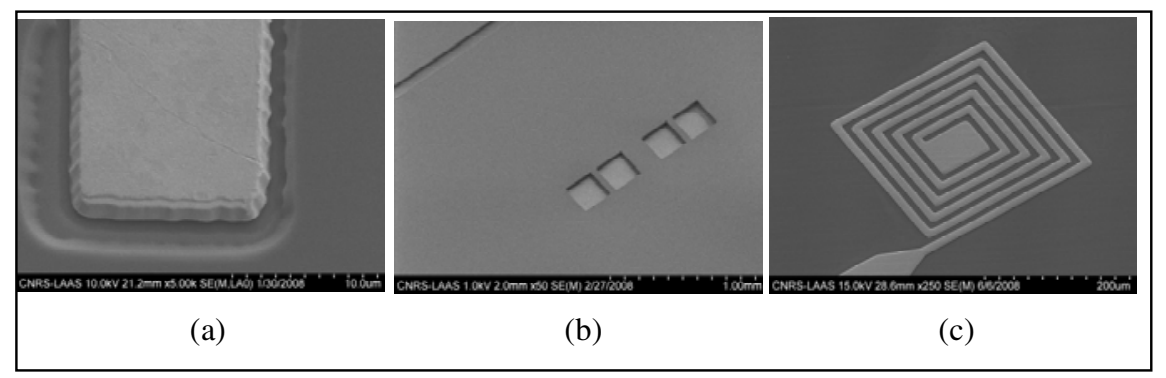

Fig. 10. SEM pictures of the different steps processing

A layer of SU-8 negative photoresist (Microchem 3005, 10 $\mu \mathrm{m}$ ) is spin-coated and patterned to form the channel bottom and insulate microcoils from flowing liquids. Microfluidic network is then fabricated: channel height and width are respectively 50 and $500 \mu \mathrm{m}$, and thickness of the channel lid is $10 \mu \mathrm{m}$. The SU-8 microfluidic network is optically transparent $(n=1.8)$ as demonstrate on Figure 11 and allows therefore optical detection and characterization of the microsystem. The resulting device was finally released from the substrate thanks to the poor adhesion of the PET layer.

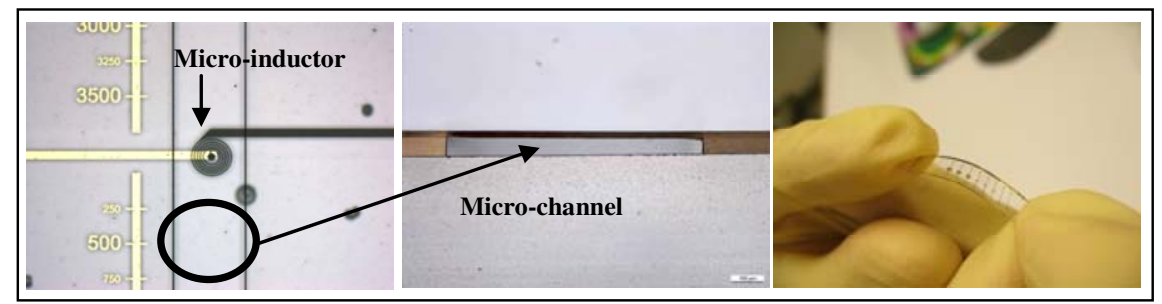

Fig. 11. Photographs of the flexible integrated microsystem with SU-8 microfluidic network

\subsection{Magnetic Microbeads Trapping}

Magnetic micro and nano-beads have proven to be very interesting and reliable tool in biological and chemical analysis in recent years. Separation or purification are often 
practiced using magnetic labeled beads in biological laboratory and sometimes for biomedical diagnosis [1, 2]. The experimental procedure for the realization of an experimental bead separation is illustrated in Figure 12. In general, bead based bioanalysis protocols use permanent magnet $[3,4]$ and repeat a specific sequence manipulation.

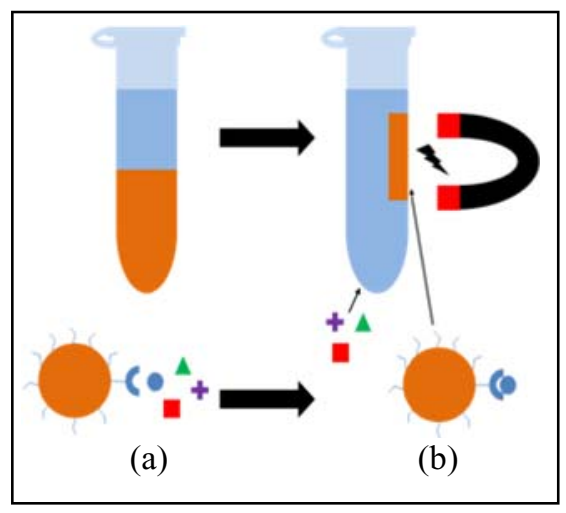

Fig. 12. Procedural sequences for magnetic bead separation. a) Magnetic bead labeled incubation with antigen target. b) Separation of magnetic bead to the solution.

In a first step, magnetic microbeads are functionalized with a probe (antibody) specific of the targeted molecule. Then, the target (antigen) contain in the sample is recognized by the probe and adsorbs specifically on the microbead surface. Finally, microbeads are trapped by an permanent magnet. The goal of our study was to implement such kind of protocol in a lab on chip device integrating a microelectromagnet. Compared to permanent magnets, electromagnets offer a higher flexibility and a higher control of magnetic field. Since small dimensions are able to be manufactured in microfluidic system, the combination of microelectromagnets and microfluidic network offers an interesting approach to this integrated microsystem $[5,6,7$ and 8$]$.

\subsection{Real Time Experimentation and Validation}

The choice of the type of magnetic microbeads depends on the application specifications. In this study, we choose to use Dynabeads® M500 Subcellular microbeads and Dynabeads® M270 Carboxylic Acid with diameter of respectively 5 $\mu \mathrm{m}$ and $2.8 \mu \mathrm{m}$. Figure 13 shows a SEM photograph of Dynabeads® M270 and an optical picture of microbeads in microfluidic channel.

In order to validate our technological concept, chips integrating microelectromagnets in a microfluidic channel have been fabricated. Magnetic bead separation has been performed using these systems. Figure 14 shows a schematic overview (a) and cross-section (b) of the system. 


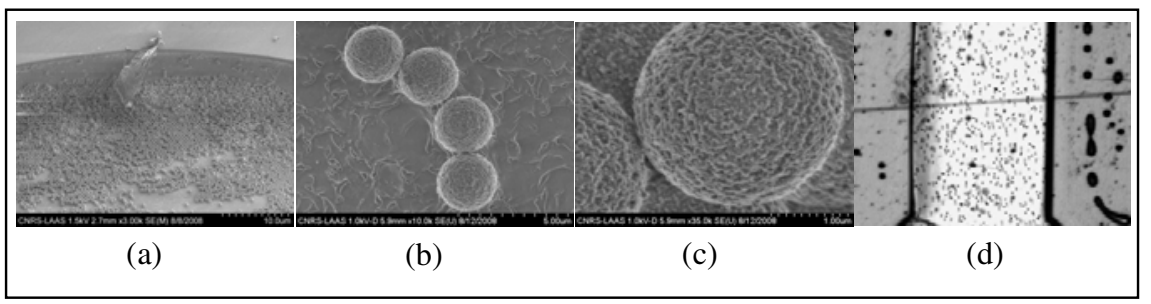

Fig. 13. SEM photographs of Dynabeads M270 (a, b and c), and optical picture of flow microbeads in micro-channels $(\mathrm{d})$

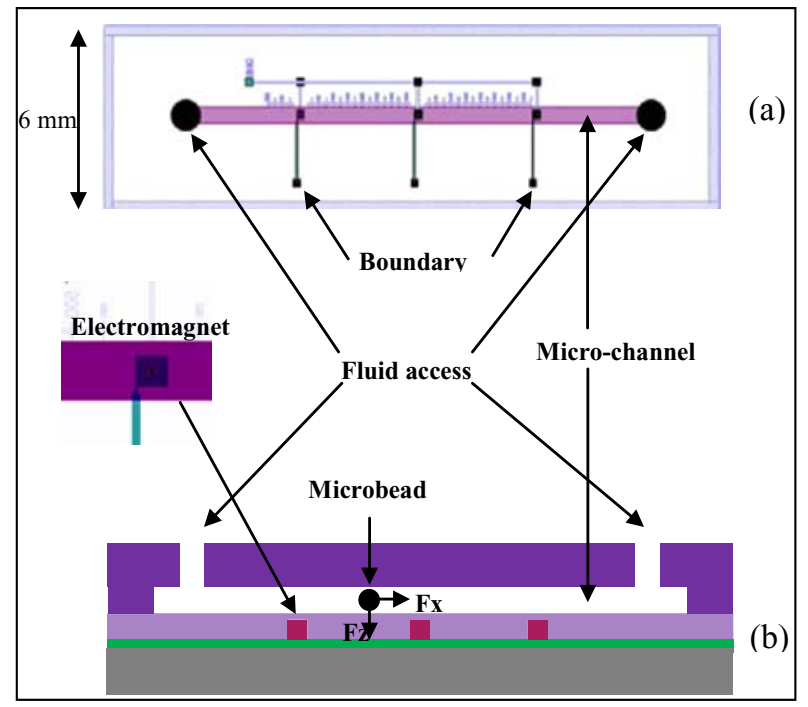

Fig. 14. The complete microsystem. a) An overview of the chips with three microelectromagnets, the micro-channel with flow inlet and outlet. b) A cross-section of the chip

The experimental setup is shown in Figure 15. The instrumental bench includes a CCD camera for recording of bead trapping, a syringe for dispensing the liquid sample and a DC power supply.

A driving current of 50mA was applied to the inductor. Experimentation were performed using a square micro-coils with 5 turns and a square section $(5 \mu \mathrm{m} * 5 \mu \mathrm{m})$.

The video frame is constituting by 12 frames per second. The main steps of trapping are reported on Figure 16:

$\Rightarrow$ Firsr we present the magnetic microbead contained in the microfluidic channel before supplying current to the microelectromagnet (0s).

$\Rightarrow$ Then, current was applied to the microelectromagnet and microbeads are trapped at the micro-channel bottom just above the micro-coils (transient analysis from 10 s to $100 \mathrm{~s}$ ). 


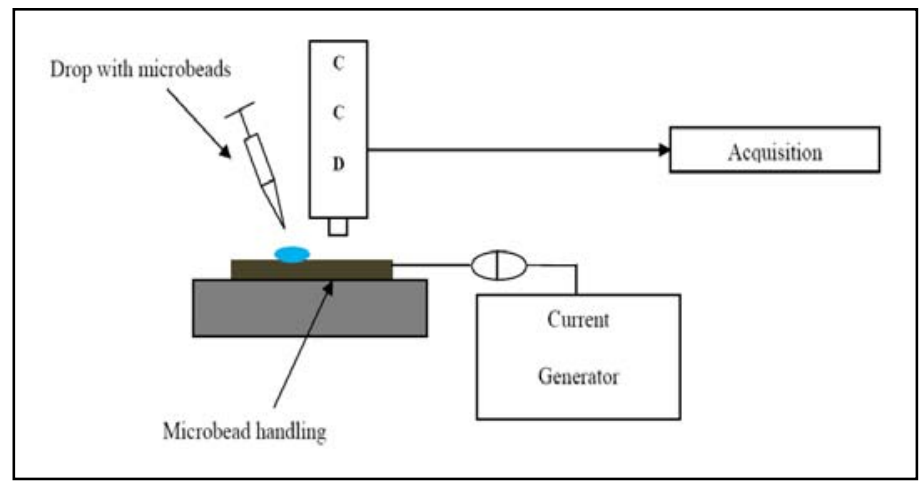

Fig. 15. Experimental setup to test and demonstrate our microbead handling

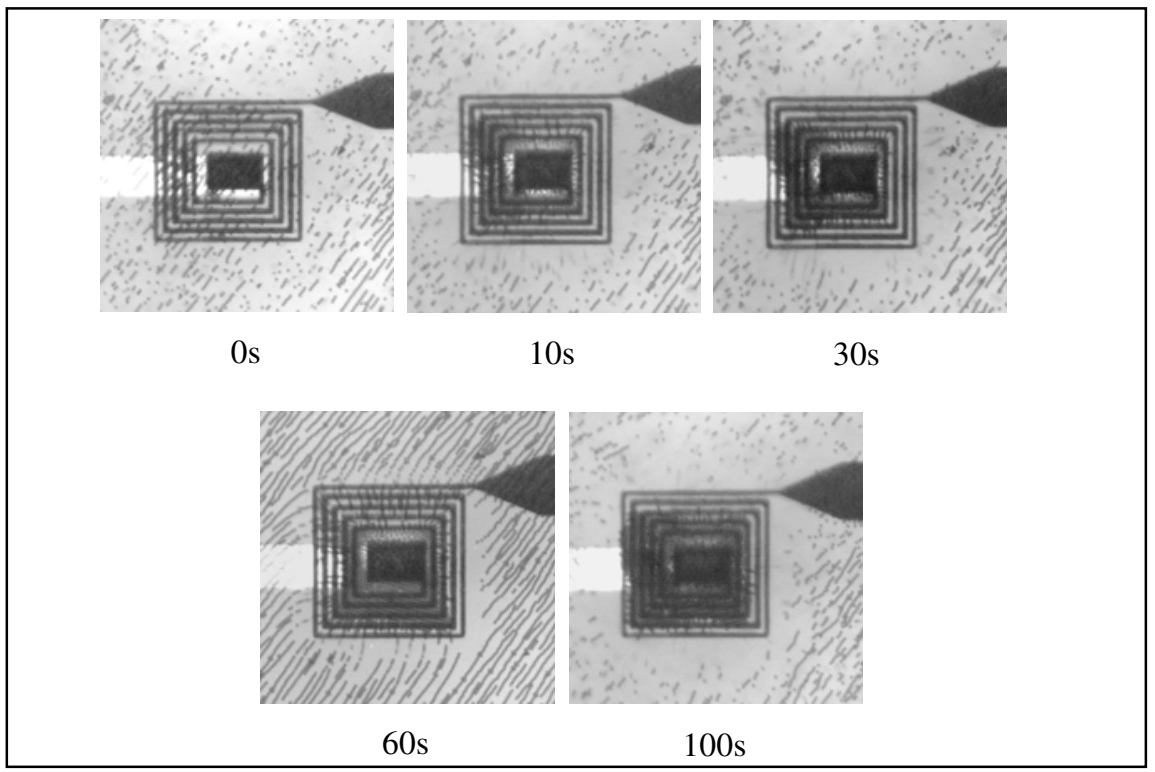

Fig. 16. Magnetic beads trapping using our microelectromagnets. (0s) without excitation applied. (10s to $100 \mathrm{~s})$ after excitation $(\mathrm{I}=50 \mathrm{~mA})$ applied to the microelectromagnet: trapping beads.

Figure 17 shows a comparison between real time magnetic beads trapping and modeling to magnetic field distribution around the microelectromagnets.

This example put the stress on the good correlation between our Finite Element approach and our experimental realization. Future work will be done to further improve the trapping and sorting efficiency of the flexible integrated microsystem by a design optimization of our microelectromagnets and microfluidic network. 


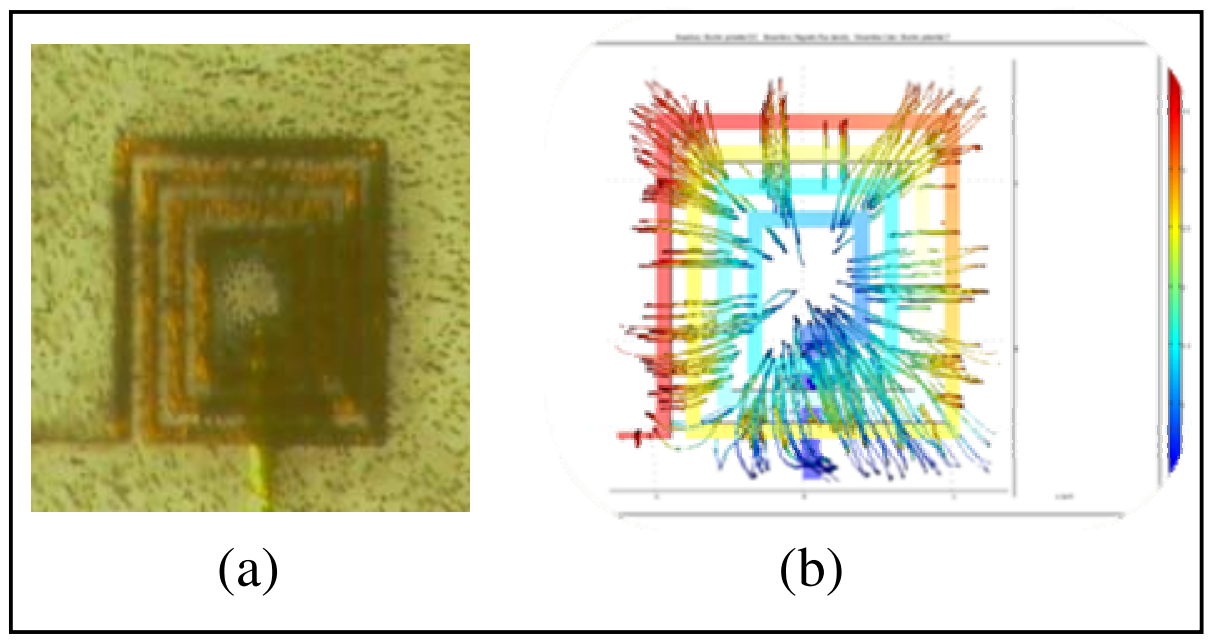

Fig. 17. Comparison between our real time experimentation and modeling: magnetic field repartition around the microelectromagnet

\section{Conclusion}

In this study, we describe magnetic field modelling created by a planar microinductor. A 3D model of the micro-spiral inductor was been realized by using our own analytical model and by using a second method based on COMSOL Multiphysics software. These two approaches allow us to check the magnetic field distribution generated by the inductor and the force exerted on magnetic bead. All these studies show that many parameters can influence the inter-action between the bead and the micro-spiral inductor. The model offer the behaviour response to any electrical current and can optimized geometrical coil parameters respect to microbeads and fluid characteristics. The first experimental results with a fluidic structure fabricated entirely in a biocompatible polymer give to our approach a novel dynamic and validate the concept and its analytical model used for actuating the biological species.

\section{References}

[1] Norgall, S., Papoutsi, M., Rôssler, J., Schweigerer, L., Wilting, J., Weich, H.A.: Elevated expression of VEGFR-3 in lymphatic endothelial cells from lymphangiomas. BMC Cancer 7, 105 (2007)

[2] Safarik, I., Safarikova, M.: Magnetic techniques for the isolation and purification of proteins. Biomagnetic Research and Technology 2, 7 (2004)

[3] Pamme, N., Eijkela, J.C.T., Manza, A.: On-chip free-flow magnetophoresis: Separation and detection of mixtures of magnetic particles in continuous flow. Journal of Magnetism and Magnetic Materials 307, 237-244 (2006) 
[4] Kim, Y.H., Hong, S., Kim, B., Yun, S., Kang, Y.R., Paek, K.K., Lee, J.W., Lee, S.H., Ju, B.K.: Droplet-based Magnetically Activated Cell Separation. In: Proceedings of the 26th Annual International Conference of the IEEE EMBS, San Francisco, CA, USA, September $1-5$ (2004)

[5] Smistrup, K., Lund-Olesen, T., Hansen, M.F.: Microfluidic magnetic separator using an array of soft magnetic elements. Journal of Applied Physics 99, 08P102 (2006)

[6] Deng, T., Whitesides, G.M.: Manipulation of magnetic microbeads in suspension using micromagnetic systems fabricated with soft lithography. Applied Physics Letters 78, 1775-1777 (2001)

[7] Ramadan, Q., Samper, V., Poenar, D., Yu, C.: On-chip micro-electromagnets for magnetic-based bio-molecules separation. Journal of Magnetism and Magnetic Materials 281, 150-172 (2004)

[8] Rida, A., Fernandez, V., Gijs, M.A.M.: Long-range transport of magnetic microbeads using simple planar coils placed in a uniform magnetostatic field. Applied Physics Letters 83, 2396 (2003) 